\title{
Expanded Disability Status Scale-Based Disability and Dental-Periodontal Conditions in Patients with Multiple Sclerosis
}

\author{
Hasan Hatipoglu ${ }^{a}$ Sibel Canbaz Kabay ${ }^{c}$ Mujgan Gungor Hatipoglu ${ }^{b}$ \\ Hilmi Ozden ${ }^{d}$
}

Departments of a Periodontology and ${ }^{b}$ Dentomaxillofacial Radiology, Faculty of Dentistry, and ${ }^{\mathrm{C}}$ Department of Neurology, Faculty of Medicine, Dumlupinar University, Kutahya, and d Department of Anatomy, Faculty of Medicine, Eskisehir Osmangazi University, Eskisehir, Turkey

\section{Key Words}

Multiple sclerosis · Oral health · Disability evaluation

\begin{abstract}
Objective: The aim of this study was to evaluate the association between different disability states in patients with multiple sclerosis (MS) as determined by the expanded disability status scale (EDSS) and dental-periodontal measures. Subjects and Methods: Eighty patients with MS (64 females and 16 males) were included in this study. Data on MS types, attack frequency, disease duration, EDSS scores and orofacial complaints prior to an MS attack were obtained from medical records. The plaque index (PI), probing depth (PD), clinical attachment level (CAL), gingival index (GI), decayedmissing-filled teeth (DMFT) index and number of present teeth were measured during one dental examination for each subject. The MS patients were divided into the following 2 groups based on their EDSS scores: low physical disability (L-DS) and high physical disability (H-DS). Differences in dental parameters between groups of low and high disability were investigated. $p<0.05$ was considered statistically significant. Results: The mean age of the participants was $38.06 \pm 10.11$ years. Age and disease duration were higher in the H-DS MS group than in the L-DS MS group $(p<$
\end{abstract}

0.05). The PI, PD and GI were higher and the number of filled teeth was lower in the H-DS MS group than in the L-DS MS group $(p<0.05)$. The EDSS scores of the H-DS MS group presented a significant correlation with the number of decayed teeth $(r=-0.548, p=0.005)$. Orofacial complaints prior to an MS attack were reported by 18 (22.5\%) patients. Conclusion: Oral measurements revealed various differences between groups of low and high disability in MS patients. In addition, some maxillofacial-oral complaints prior to an MS attack were observed.

(c) 2015 S. Karger AG, Basel

\section{Introduction}

Multiple sclerosis (MS) is a chronic, inflammatory, demyelinating, neurodegenerative disease of the central nervous system. Its aetiology is still unknown but could include genetic and environmental factors and infection [1]. Its global prevalence rate is 30 per 100,000 individuals (with a broad range from 5 to 80 ). The estimated average age of onset is 29.2 years. The female/male ratio has been reported to be 2:1 [2]. Epidemiological studies from Turkey have shown prevalence rates of 41-101.4 per 100,000 people [3,4]. MS has been classified into the

\begin{tabular}{ll}
\hline KARGER & $\begin{array}{l}\text { ○ 2015 S. Karger AG, Basel } \\
1011-7571 / 15 / 0251-0049 \$ 39.50 / 0 \quad \text { Karger }\end{array}$ \\
E-Mail karger@karger.com & $\begin{array}{l}\text { Thisis an Open Access article licensed under the terms of the } \\
\text { Creative Commons Attribution-NonCommercial 3.0 Un- } \\
\text { ported license (CC BY-NC) (www.karger.com/OA-license), } \\
\text { applicable to the online version of the article only. Distribu- } \\
\text { tion permitted for non-commercial purposes only. }\end{array}$
\end{tabular}

Dr. Mujgan Gungor Hatipoglu

Department of Dentomaxillofacial Radiology, Faculty of Dentistry

Central Campus, Dumlupinar University, Tavsanl, Yolu 10.km

TR-43100 Kutahya (Turkey)

E-Mail mujgan121@yahoo.com 
following subtypes according to disease progression patterns: relapsing-remitting MS (RRMS), progressiverelapsing MS, primary-progressive MS and secondaryprogressive MS [5]. In addition to these subtypes, the terms 'clinically isolated syndrome' [6] and 'benign MS' [7] have been used to describe possible clinical MS patterns. After a clinical diagnosis of MS according to McDonald criteria [8], the medical treatment phases are determined based on the course of the disease (acute attack, disease modification and symptomatic therapies).

The symptoms of MS vary, but weakness, fatigue, visual problems, cognitive dysfunction, ataxia and urinary and sexual dysfunctions are common [1]. Trigeminal neuralgia (TN), temporomandibular joint (TMJ) dysfunctions, facial palsy and numbness are common and clinically relevant and have been observed in the maxillofacial area [9-11].

The potential role of mercury in amalgam fillings in the development of MS and dental caries has been frequently discussed in dental-MS-related studies [12, 13]. Periodontal diseases are quite common in the general population. This disease group exhibits features of infectious, related chronic disorders. It has been suggested that periodontal diseases may be associated with diabetes mellitus, metabolic syndrome, cardiovascular diseases, pulmonary infections, rheumatoid arthritis and Alzheimer disease [14]. Few studies with different research methodologies have examined the gingival health status of patients with MS [13, 15, 16]. No differences have been found between the examined MS patients and healthy individuals $[13,16]$. An analysis of gingival health in MS patients showed that approximately one third of MS patients present healthy gingiva [15]. Recently, a link between female MS patients and chronic periodontitis was suggested [17].

The Expanded Disability Status Scale (EDSS) described by Kurtzke [18] is commonly used to evaluate the degree of disability of MS patients. EDSS scores are based on 8 functional systems (FS) and ambulation. The scale ranges from 0 (normal) to 10 (death due to MS) in 20-step scale scores (with 0.5-unit increments).

Generally, persons with disabilities at different stages present with increased problems in the oral cavity. They have difficulty performing daily oral hygiene procedures, which may lead to oral abnormalities such as a higher risk of caries and periodontal diseases [19]. Currently, the data on oral health in individuals with different degrees of MS-related disability are limited [20]. Comparison of oral hygiene habits and their possible health results in terms of normal/less disability and bedridden patients does not seem logical because it has been emphasized that the degree of disability of an MS patient should be considered when evaluating their oral health status [15]. Fragoso et al. [20] mentioned EDSS scores in their oralrelated clinical examination of MS patients. However, they did not categorize their patients into subgroups according EDSS scores. Hence, the aim of this study was to evaluate the associations between periodontal-dental measures and EDSS scores in patients with MS; in addition, the probable relation between maxillofacial and oral symptoms before an MS attack and the degree of disability was investigated.

\section{Subjects and Methods}

Included in this study were 80 patients with MS who met the revised McDonald diagnostic criteria [8] (64 females and 16 males, age range: $18-62$ years). All of the participants were recruited from the Department of Neurology of our institution. The study protocol was approved by the institutional ethics committee and used in accordance with the Declaration of Helsinki. All participants received adequate information regarding the study's design and aim and gave written informed consent.

Attention was paid to ensuring that the patients were free of an acute attack at the time of examination. Exclusion criteria were: refusal to give written consent, pregnancy or nursing, mental retardation and the need for antibiotic prophylaxis before periodontal probing.

\section{Determination of MS Status}

The neurologic examination of each patient was carried out in the Department of Neurology. EDSS scores were assigned by a neurologist (S.C.K.) with formal MS-EDSS study experience. Consistency with the existing medical records was assessed and the records were updated in case of any changes. Data for age, gender, MS type, disease duration, relapse frequency after MS diagnosis (last 2 years) and applied MS treatments were obtained from the medical records.

EDSS scores were established in accordance with Kurtzke [18]. The scale is an ordinal one (20-step scale with 0.5 -unit increments) with disease steps between 0 and 10. It measures impairment or activity limitations based on examination of the following 8 FS: pyramidal, cerebellar, brain stem, sensory, bowel and bladder, visual, cerebral and other and ambulation. EDSS steps 1.0-4.5 refer to fully ambulatory patients, and the precise step number is defined by the FS score(s), while EDSS steps 5.0-9.5 are mostly described by impairment of ambulation. Score 10 refers to death due to MS. All individuals were subgrouped with reference to EDSS scores according to disease physical disability status as indicated by a previous neurological study [21]. The cut-off value for EDSS scores was 3.5 for the subgroups. According to the related study, the subgroups were defined as follows: EDSS $\leq 3.5$ - low physical disability (L-DS), and EDSS $>3.5$ - high physical disability (H-DS).
Med Princ Pract 2016;25:49-55 DOI: $10.1159 / 000440980$
Hatipoglu/Canbaz Kabay/ Gungor Hatipoglu/Ozden 
Table 1. EDSS scores arranged by MS clinical pattern and MS therapy type

\begin{tabular}{|c|c|c|c|c|c|c|}
\hline & \multicolumn{2}{|c|}{ L-DS MS group } & \multicolumn{2}{|c|}{ H-DS MS group } & \multicolumn{2}{|c|}{ All MS participants } \\
\hline & $\mathrm{n}$ & $\%$ & $\mathrm{n}$ & $\%$ & $\mathrm{n}$ & $\%$ \\
\hline MS type & 55 & & 25 & & 80 & \\
\hline RRMS & 55 & 100 & 8 & 32 & 63 & 78.75 \\
\hline PRMS & - & - & 9 & 36 & 9 & 11.25 \\
\hline PPMS & - & - & 1 & 4 & 1 & 1.25 \\
\hline SPMS & - & - & 7 & 28 & 7 & 8.75 \\
\hline \multicolumn{7}{|l|}{ Received MS therapy } \\
\hline IFN- $\beta$ 1a & 26 & 47.3 & 3 & 12 & 29 & 36.25 \\
\hline IFN- $\beta 1 \mathrm{~b}$ & 10 & 18.2 & 5 & 20 & 15 & 18.75 \\
\hline Glatiramer acetate & 6 & 10.9 & 3 & 12 & 9 & 11.25 \\
\hline Fingolimod & 2 & 3.6 & 2 & 8 & 4 & 5 \\
\hline Natalizumab & 1 & 1.8 & 3 & 12 & 4 & 5 \\
\hline Immunosuppressant & 1 & 1.8 & 3 & 12 & 4 & 5 \\
\hline Symptomatic medical treatment & 3 & 5.5 & 4 & 16 & 7 & 8.75 \\
\hline Followed without medical treatment & 6 & 10.9 & 2 & 8 & 8 & 10 \\
\hline MS disability distribution ratio & & 68.75 & & 31.25 & & \\
\hline
\end{tabular}

PRMS = Progressive-relapsing MS; PPMS = primary-progressive MS; SPMS = secondary-progressive MS.

\section{Determination of Periodontal-Dental Status}

All periodontal and dental clinical measurements were taken by an experienced dental examiner (H.H.). Periodontal measurements were carried out with William's type manual periodontal probes. The periodontal probing depth (PD) was measured at 6 gingival sites, i.e. mesio-buccal, mid-buccal, disto-buccal, mesio-lingual, mid-lingual and disto-lingual. The PD was defined as the distance from a gingival margin to the base of the sulcus/pocket. In addition, clinical attachment levels (CAL) and the distances from the cementoenamel junction to the base of the sulcus/pocket were also recorded.

The plaque index (PI) and the gingival index (GI) were calculated for each patient to evaluate the status of dental plaque and gingival inflammation [22]. The numbers of decayed, missing and filled teeth were also recorded. Caries examinations were done using a sickle-shaped explorer. The Decayed, Missing and Filled Teeth (DMFT) index score was calculated by excluding the third molar teeth [23].

TMJ and extra-oral regional abnormalities (e.g. asymmetry and pain) were noted if present. The medical records of the patients with previous extraordinary orofacial complaints were further reviewed.

\section{Statistical Analysis}

Statistical analysis was performed using SPSS statistical software (SPSS 11.5, SPSS Inc., Chicago, Ill., USA). Means \pm SD were computed for all examined variables and all subgrouped participants. Levene's test was used to analyse the homogeneity of the variances. MS groups of low and high disability were compared using the Mann-Whitney U test or the Student t test, as appropriate. A simple correlation analysis (Pearson's correlation coefficient) was used to analyse the correlation of EDSS scores and oral parameters in MS groups of low and high disability. $\mathrm{p}<0.05$ was considered statistically significant.

Disability and Oral Health in MS Patients

\section{Results}

The mean age of the individuals was $38.06 \pm 10.11$ years (range 18-62). Based on physical disability, 55 (68.75\%) patients had L-DS MS while 25 (31.25\%) had H-DS MS. The clinical pattern and therapy of patients along with the MS types are presented in table 1 . Because 2 patients in the H-DS MS patient group were edentulous, periodontal examination was performed in $78 \mathrm{MS}$ patients.

Mean age (L-DS MS group: $36.15 \pm 9.14$; H-DS MS group: $42.28 \pm 11.02$ ) and disease duration (L-DS MS group: $5.84 \pm 5.66$; H-DS MS group: $13.08 \pm 7.47$ ) values were significantly higher in the H-DS MS group than in the L-DS MS group $(p<0.05)$. Relapse frequencies were similar in the L-DS and H-DS MS groups (table 2).

The PI (L-DS MS group: $1.07 \pm 0.83$; H-DS MS group: $1.85 \pm 1.01$ ), PD (L-DS MS group: $2.05 \pm 0.51$; H-DS MS group: $2.43 \pm 0.70$ ) and GI (L-DS MS group: $0.71 \pm 0.58$; H-DS MS group: $1.09 \pm 0.80$ ) were higher and the number of filled teeth (L-DS MS group: $3.98 \pm 4.98$; H-DS MS group: $1.36 \pm 2.88$ ) was lower in the H-DS MS group compared to the L-DS MS group ( $<0.05)$. CAL, DMFT, and the numbers of decayed teeth, missing teeth and present teeth were similar between the 2 subgroups (table 2). No significant correlation between EDSS scores and the evaluated oral parameters was observed, except for the num- 
Table 2. Clinical features of all and EDSS-subgrouped MS individuals

\begin{tabular}{|c|c|c|c|c|c|c|c|c|}
\hline & \multicolumn{2}{|c|}{ L-DS MS group } & \multicolumn{2}{|c|}{ H-DS MS group } & \multirow[t]{2}{*}{$\mathrm{p}$} & \multicolumn{3}{|c|}{ All MS participants } \\
\hline & $\mathrm{n}$ & mean $\pm \mathrm{SD}$ & $\mathrm{n}$ & mean $\pm \mathrm{SD}$ & & $\mathrm{n}$ & mean $\pm S D$ & range \\
\hline Age, years & 55 & $36.15 \pm 9.14$ & 25 & $42.28 \pm 11.02$ & $0.011^{*}$ & 80 & $38.06 \pm 10.11$ & $18-62$ \\
\hline Disease duration, years & 55 & $5.84 \pm 5.66$ & 25 & $13.08 \pm 7.47$ & $0.0001^{*}$ & 80 & $8.10 \pm 7.09$ & $1-34$ \\
\hline Relapse frequency (in the last 2 years) ${ }^{\mathrm{a}}$ & 41 & $2.00 \pm 1.22$ & 18 & $2.44 \pm 2.18$ & 0.321 & 59 & $2.14 \pm 1.57$ & $1-10$ \\
\hline \multicolumn{9}{|l|}{ Periodontal-dental parameters } \\
\hline $\mathrm{PI}^{\mathrm{b}}$ & 55 & $1.07 \pm 0.83$ & 23 & $1.85 \pm 1.01$ & $0.001^{*}$ & 78 & $1.30 \pm 0.95$ & $0-3$ \\
\hline $\mathrm{PD}^{\mathrm{b}}$ & 55 & $2.05 \pm 0.51$ & 23 & $2.43 \pm 0.70$ & $0.043^{*}$ & 78 & $2.16 \pm 0.59$ & $1-4$ \\
\hline $\mathrm{GI}^{\mathrm{b}}$ & 55 & $0.71 \pm 0.58$ & 23 & $1.09 \pm 0.80$ & $0.024^{*}$ & 78 & $0.83 \pm 0.67$ & $0-3$ \\
\hline $\mathrm{CAL}^{\mathrm{b}}$ & 55 & $2.10 \pm 0.57$ & 23 & $2.51 \pm 0.82$ & 0.054 & 78 & $2.22 \pm 0.67$ & $1-5$ \\
\hline DMFT index & 55 & $10.45 \pm 7.62$ & 25 & $11.32 \pm 9.51$ & 0.665 & 80 & $10.73 \pm 8.21$ & $0-28$ \\
\hline $\mathrm{D}$ & 55 & $1.13 \pm 1.81$ & 25 & $0.96 \pm 1.39$ & 0.684 & 80 & $1.08 \pm 1.69$ & $0-9$ \\
\hline M & 55 & $5.34 \pm 6.14$ & 25 & $9.00 \pm 8.95$ & 0.076 & 80 & $6.49 \pm 7.29$ & $0-28$ \\
\hline $\mathrm{F}$ & 55 & $3.98 \pm 4.98$ & 25 & $1.36 \pm 2.88$ & $0.024^{*}$ & 80 & $3.16 \pm 4.58$ & $0-18$ \\
\hline Present teeth & 55 & $22.65 \pm 6.14$ & 25 & $19.00 \pm 8.95$ & 0.076 & 80 & $21.51 \pm 7.29$ & $0-28$ \\
\hline
\end{tabular}

${ }^{*} \mathrm{p}<0.05 .{ }^{\mathrm{a}}$ Twenty-one patients did not have an MS relapse in the last 2 years. ${ }^{\mathrm{b}}$ Two patients in the H-DS MS group were edentulous. $\mathrm{D}=$ Decayed teeth; $\mathrm{M}=$ missing teeth; $\mathrm{F}=$ filled teeth.

ber of decayed teeth and EDSS scores in the H-DS MS patient group $(\mathrm{r}=-0.548, \mathrm{p}=0.005$; table 3$)$. Five of 80 MS patients (6.25\%) presented nonsynchronous motion, pain and clicking in the TMJ area. TN findings or facial palsy were not observed. A number of patients $(22.5 \%$, $\mathrm{n}=18)$ stated complaints prior to an MS relapse in the maxillofacial-oral area (table 4).

\section{Discussion}

In this study, CAL, DMFT, decayed teeth, missing teeth and present teeth values were similar in both L-DS and H-DS subgroups of MS patients, while PI, PD and GI indices were statistically higher in the H-DS group than in the L-DS MS group. The number of filled teeth was lower in the H-DS MS group than in the L-DS MS group. Correlation was observed between EDSS scores and the number of decayed teeth in the H-DS MS patient group. Subjective orofacial complaints were reported by a minority of the MS patients, and most of them had RRMS with low disability.

Our study is unique in its methodology since it MS patients are subgrouped according to EDSS scores. Previous studies have compared dental and/or periodontal status in MS patients with an undefined disability status (between groups or with control groups) $[10,13,15,16]$. Fragoso et al. [20] used EDSS scores in their oral-MS-
Table 3. Correlations between EDSS scores and the investigated periodontal-dental parameters in subgrouped MS patients

\begin{tabular}{|c|c|c|c|c|}
\hline & \multicolumn{2}{|c|}{ L-DS MS group } & \multicolumn{2}{|c|}{ H-DS MS group } \\
\hline & $\mathrm{r}$ & $\mathrm{p}$ & $\mathrm{r}$ & $\mathrm{p}$ \\
\hline PI-EDSS & 0.040 & 0.772 & 0.008 & 0.972 \\
\hline PD-EDSS & 0.005 & 0.969 & 0.065 & 0.767 \\
\hline GI-EDSS & 0.025 & 0.856 & 0.091 & 0.681 \\
\hline CAL-EDSS & -0.016 & 0.909 & 0.086 & 0.698 \\
\hline DMFT-EDSS & 0.086 & 0.532 & 0.155 & 0.458 \\
\hline D-EDSS & 0.125 & 0.363 & -0.548 & $0.005^{*}$ \\
\hline M-EDSS & 0.033 & 0.811 & 0.250 & 0.228 \\
\hline F-EDSS & 0.045 & 0.743 & 0.002 & 0.994 \\
\hline Present teeth-EDSS & -0.033 & 0.811 & -0.250 & 0.228 \\
\hline
\end{tabular}

${ }^{*} \mathrm{p}<0.05 . \mathrm{D}=$ Decayed teeth; $\mathrm{M}=$ missing teeth; $\mathrm{F}=$ filled teeth.

related study. Nevertheless, they did not categorize their patients according to EDSS scores.

The DMFT index has been used to evaluate dental status in MS patients in previous studies, and the results have been reported [10, 13, 15, 16]. Kovac et al. [10] and Symons et al. [16] observed no differences in the DMFT index between the MS and control groups. We found that DMFT, decayed teeth and missing teeth scores and the number of present teeth were similar between the L-DS MS and H-DS MS groups. The number of filled teeth in the H-DS MS 
Table 4. Subjective symptom(s) prior to an MS relapse in the maxillofacial and oral area as reported by the individuals

\begin{tabular}{llll}
\hline $\begin{array}{l}\text { Patient } \\
\text { No. }\end{array}$ & MS type & EDSS score & Subjective maxillofacial and oral symptom(s) \\
\hline 1 & RRMS & 1.0 & Significant increase in dry mouth feeling \\
2 & RRMS & 1.5 & Change in sense of taste \\
3 & RRMS & 1.0 & Significant increase in dry mouth feeling \\
4 & RRMS & 1.5 & Change in sense of taste \\
5 & RRMS & 1.0 & Canker sore(s) \\
6 & RRMS & 2.0 & Watery mouth feeling/difficulty swallowing \\
7 & PRMS & 5.0 & Canker sore(s) \\
8 & SPMS & 7.5 & Significant increase in dry mouth feeling \\
9 & RRMS & 1.0 & Significant increase in dry mouth feeling \\
10 & RRMS & 3.0 & Significant increase in dry mouth feeling/difficulty swallowing \\
11 & RRMS & 2.0 & Change in sense of taste \\
12 & RRMS & 1.0 & Palate/gingival swelling sensation \\
13 & RRMS & 1.5 & Significant increase in dry mouth feeling/difficulty swallowing \\
14 & RRMS & 1.0 & Lip-tongue-facial numbness \\
15 & RRMS & 0.0 & Tongue numbness (in the tip region) \\
16 & RRMS & 2.5 & Significant increase in dry mouth feeling/burning sensation \\
17 & RRMS & 2.5 & Significant increase in dry mouth feeling/change in sense of taste \\
18 & RRMS & 1.0 & Significant increase in dry mouth feeling and halitosis \\
\hline
\end{tabular}

PRMS = Progressive-relapsing MS; SPMS = secondary-progressive MS.

group was lower compared to the L-DS MS group. Different methods have been used in previous studies of periodontal status evaluation $[13,16,17]$. In the current study, we measured the PI, GI, PD and CAL to evaluate periodontal health in detail. Thus, it is difficult to compare previous studies with each other due to differences in study design and methodology variances. McGrother et al. [13] and Symons et al. [16] found similar results on gingival health between MS patients and controls. In this study, PI, PD and GI indices were statistically higher in the H-DS MS group.

These controversial results may be due to two reasons. Firstly, the small sample size of these previous studies may be the principal limitation for analysis; a second limitation may be inadequate grouping of MS patients according to the evaluated parameters. In the current study, we evaluated MS patients grouped according to physical disability status, and a larger sample size was available. Thus, some controversial issues with previous studies have been improved upon.

The primary limitation of the current study comes from intrinsic issues of EDSS in evaluating the disability status of MS patients. The scale is not linear, it overemphasizes ambulation, and it has problems of reliability that need to be discussed. However, these discussions are not in the scope of the current study. Kalkers et al. [24] discussed various issues of EDSS and a different scale, i.e. the MS Functional Composite (MSFC) scale. They disputed that the MSFC scale supplies 3-dimensional quantitative information including arm and hand function. Thus, these issues should be considered in the design of other specific oral health studies in MS. Another limitation which may be mentioned is the lack of a control group in the current study.

We observed some additional oral symptoms according to the medical records, such as a change in the sense of taste, a burning sensation or an increased dry mouth feeling in some patients prior to an MS relapse. These findings were common among patients with RRMS with L-DS. Orofacial symptoms/complaints may be considered signs of a possible MS relapse, especially in RRMS patients with L-DS. Pain, numbness, TN, TMJ complaints, facial palsy and dysphagia have been reported in MS patients [10, 11, 25, 26]. A small proportion of our patients (5 of $80,6.25 \%$ ) had TMJ complaints. TN or facial palsy was not observed.

Another subject to be emphasized is drug use in MS patients. This is an underrated and difficult factor to eliminate in clinical MS-oral related research. The use of certain drugs may be continuous. Corticosteroids and disease-modifying drugs [like interferon (IFN)- $\beta$ drugs, 
glatiramer acetate and natalizumab] are commonly used in MS $[11,27]$. Moreover, some drugs (such as antidepressants, anticonvulsants and antispasmodics) control MS-related symptoms alone or in combination with others [11]. Corticosteroid-related alterations in the periodontium have been discussed previously $[28,29]$. IFN may participate in periodontal inflammation in response to bacterial components or products [30]. The existing literature on the disease-modifying drugs used for MS supplies little information about their possible clinical effects on oral-periodontal tissues. We observed that only $10 \%$ of the study population was followed without a drug treatment. The main purpose of this study was to evaluate the associations between dentalperiodontal measures and disability severity in MS patients. It was not our intention in the current study to evaluate drug-related oral alterations. In addition, the mismatched numbers of patients on different drugs limited our ability to compare drug-based groups. It was not possible for us to draw conclusions on drug-related issues. The clinical effects of MS medications on the oral cavity, especially periodontal tissues, are worthy of further studies.
This is a preliminary study. Thus, the results should not be generalized due to convenient sampling. Our study method and results indicate that dental-periodontal studies on MS should have a wide perspective and should also focus on different MS types, MS disease duration, MS relapse frequency and even the specific drugs used for MS. This approach has the potential to improve our basic knowledge of dental-periodontal diseases and their relation to MS.

\section{Conclusion}

PI, PD and GI indices were higher in the H-DS MS group than in the L-DS MS group. The number of filled teeth was lower in the H-DS MS group than in the L-DS MS group. In addition, a number of patients was observed to have maxillofacial-oral complaints prior to an attack, especially patients with the RRMS type and L-DS. These observations merit further clinical observations in order to understand dental-periodontal disease formation and progression in MS patients.

\section{References}

1 National Institutes of Health - National Institute of Neurological Disorders and Stroke: Multiple Sclerosis: Hope Through Research. 2014. http://www.ninds.nih.gov/disorders/ multiple_sclerosis/detail_multiple_sclerosis. htm.

2 World Health Organization, Multiple Sclerosis International Federation: Atlas Multiple Sclerosis Resources in the World. 2008. http:// www.who.int/mental_health/neurology/Atlas_MS_WEB.pdf.

-3 Türk Börü U, Alp R, Sur H, et al: Prevalence of multiple sclerosis door-to-door survey in Maltepe, Istanbul, Turkey. Neuroepidemiology 2006;27:17-21.

4 Börü UT, Tasdemir M, Güler N, et al: Prevalence of multiple sclerosis: door-to-door survey in three rural areas of coastal Black Sea regions of Turkey. Neuroepidemiology 2011; 37:231-235.

5 Lublin FD, Reingold SC: Defining the clinical course of multiple sclerosis: results of an international survey. Neurology 1996;46:907911.

6 Miller DH, Chard DT, Ciccarelli O: Clinically isolated syndromes. Lancet Neurol 2012;11: 157-169.
7 Correale J, Ysrraelit MC, Fiol MP: Benign multiple sclerosis: does it exist? Curr Neurol Neurosci Rep 2012;12:601-609.

8 Polman CH, Reingold SC, Banwell B, et al: Diagnostic criteria for multiple sclerosis: 2010 revisions to the McDonald criteria. Ann Neurol 2011;69:292-302.

-9 Chemaly D, Lefrançois A, Pérusse R: Oral and maxillofacial manifestations of multiple sclerosis. J Can Dent Assoc 2000;66:600-605.

10 Kovac Z, Uhac I, Buković D, et al: Oral health status and temporomandibular disorders in multiple sclerosis patients. Coll Antropol 2005;29:441-444.

11 Fischer DJ, Epstein JB, Klasser G: Multiple sclerosis: an update for oral health care providers. Oral Surg Oral Med Oral Pathol Oral Radiol Endod 2009;108:318-327.

$>12$ Huggins HA, Levy TE: Cerebrospinal fluid protein changes in multiple sclerosis after dental amalgam removal. Altern Med Rev 1998;3:295-300.

13 McGrother CW, Dugmore C, Phillips MJ, et al: Multiple sclerosis, dental caries and fillings: a case-control study. Br Dent J 1999;187: 261-264.
14 Pizzo G, Guiglia R, Lo Russo L, et al: Dentistry and internal medicine: from the focal infection theory to the periodontal medicine concept. Eur J Intern Med 2010;21:496-502.

15 Santa Eulalia-Troisfontaines E, MartínezPérez EM, Miegimolle-Herrero M, et al: Oral health status of a population with multiple sclerosis. Med Oral Patol Oral Cir Bucal 2012; 17:e223-e227.

16 Symons AL, Bortolanza M, Godden S, et al: A preliminary study into the dental health status of multiple sclerosis patients. Spec Care Dentist 1993;13:96-101.

$\checkmark 17$ Sheu JJ, Lin HC: Association between multiple sclerosis and chronic periodontitis: a population-based pilot study. Eur J Neurol 2013; 20:1053-1059.

18 Kurtzke JF: Rating neurologic impairment in multiple sclerosis: an Expanded Disability Status Scale (EDSS). Neurology 1983;33: 1444-1452.

19 Nitschke I, Kaschke I: Zahnmedizinische Betreuung von Pflegebedürftigen und Menschen mit Behinderungen. Bundesgesundheitsblatt Gesundheitsforschung Gesundheitsschutz 2011;54:1073-1082. 
20 Fragoso YD, Alves HHC, Alves LC, et al: Dental care in multiple sclerosis: an overlooked and under-assessed condition. J Disabil Oral Health 2010:11:53-56.

21 Russo C, Morabito F, Luise F, et al: Hyperhomocysteinemia is associated with cognitive impairment in multiple sclerosis. J Neurol 2008;255:64-69.

22 Löe H: The gingival index, the plaque index and the retention index systems. J Periodontol 1967;38:610-616.

23 Hiremath SS: Indices; in Hiremath SS (ed): Text Book of Preventive and Community Dentistry. New Delhi, Elsevier, 2007, pp 180200.
24 Kalkers NF, Polman CH, Uitdehaag BMJ: Measuring clinical disability: the MS functional composite. Int MS J 2001;8:78-87.

25 Danesh-Sani SA, Rahimdoost A, Soltani M, et al: Clinical assessment of orofacial manifestations in 500 patients with multiple sclerosis. J Oral Maxillofac Surg 2013;71:290-294.

26 Fernandes AM, Duprat AD, Eckley CA, et al: Oropharyngeal dysphagia in patients with multiple sclerosis: do the disease classification scales reflect dysphagia severity? Braz J Otorhinolaryngol 2013;79:460-465.

27 Alroughani R, Al Hashel J, Thussu A, et al: Use of natalizumab in patients with active relapsing-remitting multiple sclerosis in $\mathrm{Ku}$ wait. Med Princ Pract 2013;22:495-499.
28 Seymour RA: Effects of medications on the periodontal tissues in health and disease. Periodontol 2000 2006;40:120-129.

29 Safkan B, Knuuttila M: Corticosteroid therapy and periodontal disease. J Clin Periodontol 1984;11:515-522.

- 30 Sakuta T, Tokuda M, Tamura M, et al: Dual regulatory effects of interferon-alpha, -beta, and -gamma on interleukin- 8 gene expression by human gingival fibroblasts in culture upon stimulation with lipopolysaccharide from Prevotella intermedia, interleukin-1 alpha, or tumor necrosis factor-alpha. J Dent Res 1998;77:1597-1605. 